\title{
Utility of CD200 immunostaining in the diagnosis of primary mediastinal large B cell lymphoma: comparison with MAL, CD23, and other markers
}

\author{
David M Dorfman ${ }^{1,2}$, Aliakbar Shahsafaei ${ }^{1}$ and Miguel A Alonso ${ }^{3}$ \\ ${ }^{1}$ Department of Pathology, Brigham and Women's Hospital, Boston, MA, USA; ${ }^{2}$ Department of Pathology, \\ Harvard Medical School, Boston, MA, USA and ${ }^{3}$ Centro de Biologia Molecular 'Severo Ochoa,' Co1nsejo \\ Superior de Investigaciones Cientificas, Universidad Autonoma de Madrid, Madrid, Spain
}

\begin{abstract}
CD200, an immunoglobulin superfamily membrane glycoprotein, is expressed in a number of B cell lymphoproliferative disorders, including primary mediastinal large B cell lymphoma, but not diffuse large B cell lymphoma, based on a preliminary study. Here, we compare the expression of CD200 with other markers of primary mediastinal large B cell lymphoma, including MAL and CD23, in formalin-fixed, paraffin-embedded histologic sections from a series of $\mathbf{3 5}$ cases of primary mediastinal large B cell lymphoma and $\mathbf{3 0}$ cases of diffuse large B cell lymphoma. CD200 exhibits the greatest staining sensitivity of the markers studied: $94 \%$, compared with CD23 (69\%), MAL (86\%), TRAF (86\%), and REL (77\%). It exhibits staining specificity of $93 \%$, similar to that of CD23 (93\%) and MAL (97\%), and greater than that of TRAF (77\%) and REL (83\%). We conclude that CD200 is a practical and useful marker for the diagnosis of primary mediastinal large B cell lymphoma. Modern Pathology (2012) 25, 1637-1643; doi:10.1038/modpathol.2012.129; published online 17 August 2012
\end{abstract}

Keywords: diffuse large B cell lymphoma; REL; TRAF

CD200 or OX-2 antigen is an immunoglobulin superfamily membrane glycoprotein ${ }^{1}$ widely expressed in multiple cell types, including B cells, a subset of $\mathrm{T}$ cells, and a number of non-hematopoietic cells. ${ }^{2}$ It interacts with CD200R, a receptor expressed primarily on myeloid/monocyte lineage cells, and has a suppressive effect on $\mathrm{T}$ cell mediated immune response. ${ }^{3,4}$ CD200 is expressed in a number of hematopoietic neoplasms, including acute myeloid leukemia, ${ }^{5}$ multiple myeloma, ${ }^{6}$ and is a marker of poor prognosis when expressed in these neoplasms, probably through its immunosuppressive effect on the host immune system.

We and others have found that CD200 is expressed in a number of $\mathrm{B}$ cell-derived neoplasms, including chronic lymphocytic leukemia/small lymphocytic lymphoma, hairy cell leukemia, B lymphoblastic leukemia/ lymphoma and classical Hodgkin lymphoma, ${ }^{7,8}$ and by

Correspondence: Dr DM Dorfman, MD, PhD, Department of Pathology, Brigham \& Women's Hospital, 75 Francis Street, Boston, MA 02115, USA.

Email: ddorfman@partners.org

Received 25 May 2012; revised 13 June 2012; accepted 23 June 2012; published online 17 August 2012 follicular helper $\mathrm{T}$ cells and in a $\mathrm{T}$ cell neoplasm, angioimmunoblastic T cell lymphoma. ${ }^{9}$ In our study of CD200 expression in B cell lymphoproliferative disorders, we found that CD200 is expressed by the neoplastic B cells in primary mediastinal large B cell lymphoma (8 of 8 cases), but not in diffuse large B cell lymphoma (0 of 12 cases; $P<0.0001 ; 8$ ).

Primary mediastinal large $\mathrm{B}$ cell lymphoma, a large B cell lymphoma, is thought to arise from thymic B cells in the anterior mediastinum. It occurs in young adults with female predominance. Primary mediastinal large B cell lymphoma expresses pan-B cell markers, with variable expression of CD30, Bcl-2, Bcl-6, and CD10. A number of immunophenotypic markers of primary mediastinal large $\mathrm{B}$ cell lymphoma (to distinguish it from diffuse large B cell lymphoma) have been described, including CD23, a transmembrane glycoprotein expressed in mature B cells and follicular dendritic cells, MAL, a membrane glycoprotein expressed in thymocytes and thymic medullary B cells, TRAF and REL, nuclear factor $\kappa \mathrm{B}$ signaling pathway molecules recently identified as markers of primary mediastinal large B cell lymphoma by gene expression array analysis. All of these markers have analytical 
or practical limitations, including moderate sensitivity and specificity or lack of commercial reagent availability (10-12; summarized in Table 1).

Based on our preliminary CD200 findings, we studied a large series of cases of primary mediastinal large B cell lymphoma and diffuse large B cell lymphoma to assess the sensitivity and specificity of CD200 as an immunophenotypic marker of primary mediastinal large B cell lymphoma compared with diffuse large B cell lymphoma. We also compared its expression with that of other reported markers of primary mediastinal large $\mathrm{B}$ cell lymphoma, to assess the relative diagnostic utility of CD200.

\section{Materials and methods}

\section{Case Selection}

Cases were obtained from the files of the Department of Pathology at Brigham and Women's Hospital, Boston, MA, with the institutional internal review board's approval. The pathologic diagnoses were originally established according to the criteria of the 2008 WHO classification, based on morphologic and immunohistochemical findings. ${ }^{13}$ Cases of diffuse large B cell lymphoma exhibited a diffuse proliferation of large neoplastic lymphoid cells with nuclear size greater than that of a macrophage nucleus. Within lymphoid tissue, there was complete or partial effacement of nodal architecture, and at extranodal sites, sheets of large lymphoid cells were observed. Neoplastic cells exhibited immunostaining for one or more pan-B cell markers, including CD20, CD79a, and Pax-5/BSAP. Cases of primary mediastinal large B cell lymphoma typically presented with a large anterior mediastinal mass consisting of intermediate to large-sized lymphoid cells with round to oval nuclei with associated fibrosis. Neoplastic cells exhibited immunostaining for one or more pan-B cell markers, including CD20, CD79a, and Pax-5/ BSAP. Diagnostic slides from all cases were reviewed by one of the authors of this study (DMD).

\section{Immunohistochemistry}

Immunohistochemistry was performed on $5 \mu \mathrm{m}$ thick formalin-fixed, paraffin-embedded tissues sec- tions using a standard indirect avidin-biotin horseradish peroxidase method and diaminobenzidine color development, as previously described. ${ }^{14}$ Briefly, slides were soaked in xylene, passed through graded alcohols and put in distilled water. The sections were blocked for peroxidase activity with $3 \%$ hydrogen peroxide in ethanol for $15 \mathrm{~min}$ and washed under the running water for $5 \mathrm{~min}$. Slides were then pretreated with $10 \mathrm{~mm}$ citrate, $\mathrm{pH}$ 6.0 in a pressure cooker for $30 \mathrm{~min}$ in preheated buffer, followed by washing in distilled water. All further steps were performed at room temperature in a hydrated chamber.

Immunostaining with goat anti-human CD200 polyclonal antibody (R\&D Systems, Minneapolis, $\mathrm{MN}$ ) was compared with that of goat IgG control antibody diluted to identical protein concentration, to confirm staining specificity, as previously described. ${ }^{8,9}$ MAL antibody was previously described, ${ }^{10}$ and staining was compared with that of mouse IgG control antibody diluted to identical protein concentration, to confirm staining specificity. For cases lacking prior immunostaining results for CD23, TRAF, and REL, immunostaining was performed using mouse monoclonal antibodies obtained from The Binding Site, Birmingham, AL; Santa Cruz Biotechnology, Santa Cruz, CA; and Calbiochem, San Diego, CA, respectively, and compared with mouse IgG control antibody diluted to identical protein concentration, to confirm staining specificity.

\section{Case Evaluation}

Reactivity for CD200, CD23, TRAF, REL, and MAL was considered as positive if there was more than focal staining of neoplastic cells ( $>20 \%$ ). Regarding CD200 staining, staining was considered as homogenous if $>80 \%$ of the neoplastic cells exhibited staining, and it was considered as heterogenous if $20-80 \%$ of the neoplastic cells exhibited staining. Photomicrographs were generated on an Olympus BH2 microscope (Olympus, Center Valley, PA) using an Olympus DP25 digital camera and analyzed with the software Adobe Photoshop 11.0 (Adobe, San Jose, CA).

Table 1 Summary of immunophenotypic markers of primary mediastinal large B cell lymphoma

\begin{tabular}{|c|c|c|c|c|}
\hline Marker & $\begin{array}{c}\text { Expression in primary mediastinal } \\
\text { large B cell lymphoma }\end{array}$ & $\begin{array}{c}\text { Expression in diffuse large } \\
\text { B cell lymphoma }\end{array}$ & $\begin{array}{l}\text { Commercially } \\
\text { available? }\end{array}$ & Reference \\
\hline CD200 (preliminary study) & $8 / 8(100 \%)$ & $0 / 12(0 \%)$ & Yes & 9 \\
\hline CD200 (current study) & $33 / 35(94 \%)$ & $2 / 30(7 \%)$ & Yes & Current study \\
\hline MAL & $21 / 33(70 \%)$ & $1 / 33(3 \%)$ & No & 10 \\
\hline CD23 & $17 / 24(71 \%)$ & $12 / 100(12 \%)$ & Yes & 11 \\
\hline TRAF & $48 / 78(62 \%)$ & $20 / 173(12 \%)$ & Yes & 12 \\
\hline REL & $31 / 48(65 \%)$ & $28 / 160(18 \%)$ & Yes & 12 \\
\hline
\end{tabular}




\section{Results}

A total of 35 cases of primary mediastinal large B cell lymphoma and 30 cases of diffuse large B cell lymphoma were evaluated for expression of CD200 and other markers of primary mediastinal large B cell lymphoma. Patients with primary mediastinal large B cell lymphoma (18 women, 17 men) ranged in age from 21 to 73 years (mean $38 \pm 12$ years). Thirty-four patients presented with anterior mediastinal masses and underwent biopsy of the mass, including two needle biopsies. In two cases, patients underwent needle biopsies of the lung. One patient underwent biopsy of a chest wall mass. The neoplastic cells were typically medium to large in size with round to oval nuclei, pale cytoplasm and associated fibrosis (Figure 1a). Routine immunohistochemical staining performed as part of the diagnostic evaluation revealed that the neoplastic cells were immunoreactive for pan-B cell markers in all cases, with co-expression of CD30 in 21/29 cases, of which 13 exhibited subset staining and 5 exhibited focal staining. Bcl-2 expression was seen in 19/21 cases, of which 7 exhibited subset staining and one exhibited focal staining. Bcl-6 expression was seen in 26/28 cases, of which 9 exhibited subset staining and 2 exhibited variable staining. CD10 was expressed by 4/24 cases, with 1 exhibiting weak staining and 1 exhibiting rare staining. The Ki-67 proliferation index ranged from 20 to $95 \%$ (mean $61.0 \pm 23.5$ ).

Patients with diffuse large B cell lymphoma (19 women, 11 men) ranged in age from 34 to 85 years (mean $62 \pm 11$ years). Patients presented with lymphadenopathy in 14 cases, including 2 involving the mediastinum, and with masses at various anatomic sites in the remainder. Five of the patients underwent needle biopsies. The neoplastic cells were large in size with round to oval to irregular vesicular nuclei with distinct to prominent nucleoli and scant to moderate cytoplasm. Routine immunohistochemical staining performed as part of the diagnostic evaluation revealed that the neoplastic cells were immunoreactive for pan-B cell markers in all cases with co-expression of CD30 in 1/2 cases, Bcl-2 in 16/ 17 cases ( 3 with subset staining), Bcl-6 in 18/21 cases (1 with subset staining), and CD10 in 15/27 cases (1 with subset staining and 1 with weak staining). The Ki-67 proliferation index ranged from 20 to $95 \%$ (mean $72.1 \pm 21.7$ ).

The majority of primary mediastinal large B cell lymphoma cases exhibited immunostaining for CD200 (33/35; 94\% sensitivity; summarized in Table 2), typically with a strong membrane and cytoplasmic staining pattern (Figure 1b). Staining was homogenous in 18 cases, including 3 of the needle biopsies and heterogenous in 15 cases, including 1 of the needle biopsies. Two cases of primary mediastinal large B cell lymphoma were CD200 negative, but positive for at least two other markers of primary mediastinal large B cell lymphoma (Table 2). The patients were 44- and 73-year-old men and their neoplasms were negative for CD10 and CD30 and positive for BCL-6 (data not shown). In contrast, 28 of 30 cases of diffuse large B cell lymphoma did not exhibit immunostaining for CD200 (93\% specificity; $P<0.0001$; summarized in Table 3). Both CD200positive cases exhibited necrosis, one focal and one extensive, and were immunoreactive for one other marker of primary mediastinal large B cell lymphoma (Table 3). One biopsy specimen exhibited homogenous staining for CD200, and the other, a needle biopsy, exhibited heterogenous CD200 staining. The patients were 54 - and 85 -year-old men with CD10-negative diffuse large B cell lymphoma of the tonsil and psoas muscle, respectively.

We studied several additional markers of primary mediastinal large B cell lymphoma to compare CD200 staining results with that of other markers of primary mediastinal large B cell lymphoma. CD23 exhibited strong membrane and cytoplasmic staining in 24/35 cases of primary mediastinal large B cell lymphoma (69\% sensitivity; Figure 1c). Ten of eleven CD23-negative cases were positive for CD200, and all were positive for at least two additional markers of primary mediastinal large B cell lymphoma (Table 2). The CD23-negative cases did not exhibit any specific staining pattern for CD30, CD10, Bcl-2, and Bcl-6, although all were non-immunoreactive for CD10 (7/7 and data not shown). A total of 2 of 30 cases $(7 \%)$ of diffuse large B cell lymphoma were CD23 positive. Both cases were CD200 negative, exhibited staining for at least one additional marker of primary mediastinal large B cell lymphoma (Table 3), and were immunoreactive for Bcl-6.

MAL exhibited strong membrane and cytoplasmic staining in $30 / 35$ cases ( $86 \%$ ) of primary mediastinal large B cell lymphoma (Figure 1d). Four of five MAL-negative cases were immunoreactive for CD200 and all were immunoreactive for at least two additional markers of primary mediastinal large B cell lymphoma (Table 2). The MAL-negative cases did not exhibit any specific staining pattern for CD30, CD10, Bcl-2, and Bcl-6 (data not shown). Only 1 of the 30 diffuse large B cell lymphoma cases (3\%) was MAL positive. This case was CD200 negative, exhibited staining for two other markers of primary mediastinal large B cell lymphoma (Table 3), and was of follicular origin (immunoreactive for CD10, Bcl-6, and Bcl-2).

We found that TRAF exhibited cytoplasmic staining in 30 of 35 cases of primary mediastinal large B cell lymphoma (86\%; Figure 1e). All five TRAFnegative neoplasms were immunoreactive for CD200 and at least one additional marker of primary mediastinal large B cell lymphoma (Table 2). The TRAF-negative cases did not exhibit any specific staining pattern for CD30, CD10, Bcl-2, and Bcl-6, although all exhibited subset staining for CD30 (data not shown). In contrast, 7 of 30 cases of diffuse large B cell lymphoma (23\%) were immunoreactive for TRAF, and, in 4/7 cases, at least 1 additional 

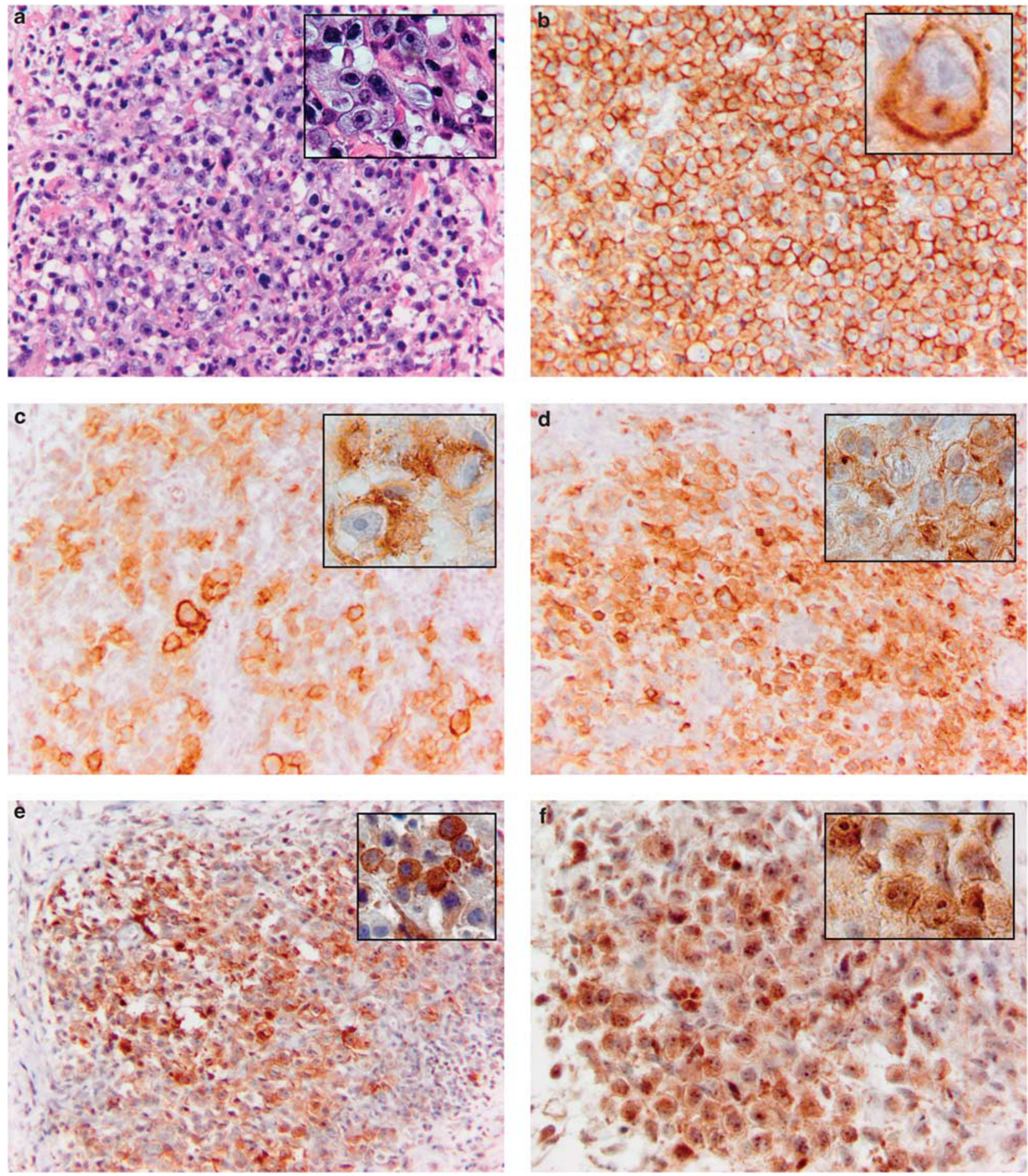

Figure 1 Primary mediastinal large B cell lymphoma (a) contains sheets of medium to large-sized neoplastic cells with round to oval nuclei, pale cytoplasm and associated fibrosis. The neoplastic cells exhibit membrane and cytoplasmic staining for CD200 (b), membrane and cytoplasmic staining for CD23 (c), membrane and cytoplasmic staining for MAL (d), primarily cytoplasmic staining for TRAF (e), and nuclear and cytoplasmic staining for REL $(\mathbf{f})$. $(\mathbf{a}-\mathbf{e}) \times 200$, $(\mathbf{f}) \times 500$; insets $\times 1000$.

primary mediastinal large B cell lymphoma marker (Table 3). All cases studied were immunoreactive for BCL-6 (6/6) and Bcl-2 (4/4). REL exhibited nuclear and cytoplasmic staining in 27 of 35 cases of primary mediastinal large B cell lymphoma (77\%; Figure 1f). Seven of eight REL-negative neoplasms were immunoreactive for CD200 and all were immunoreactive for at least 3 additional markers 
Table 2 Summary of primary mediastinal large B cell lymphoma immunostaining results. ${ }^{\text {a }}$

\begin{tabular}{|c|c|c|c|c|c|}
\hline Case no. & CD200 & CD23 & MAL & TRAF & REL \\
\hline 1 & & & & & \\
\hline 2 & & & & & \\
\hline 3 & & & & & \\
\hline 4 & & & & & \\
\hline 5 & & & & & \\
\hline 6 & & & & & \\
\hline 7 & & & & & \\
\hline 8 & & & & & \\
\hline 9 & & & & & \\
\hline 10 & & & & & \\
\hline 11 & & & & & \\
\hline 12 & & & & & \\
\hline 13 & & & & & \\
\hline 14 & & & & & \\
\hline 15 & & & & & \\
\hline 16 & & & & & \\
\hline 17 & & & & & \\
\hline 18 & & & & & \\
\hline 19 & & & & & \\
\hline 20 & & & & & \\
\hline 21 & & & & & \\
\hline 22 & & & & & \\
\hline 23 & & & & & \\
\hline 24 & & & & & \\
\hline 25 & & & & & \\
\hline 26 & & & & & \\
\hline 27 & & & & & \\
\hline 28 & & & & & \\
\hline 29 & & & & & \\
\hline 30 & & & & & \\
\hline 31 & & & & & \\
\hline 32 & & & & & \\
\hline 33 & & & & & \\
\hline 34 & & & & & \\
\hline 35 & & & & & \\
\hline sensitivity & $\begin{array}{l}33 / 35= \\
94 \%\end{array}$ & $\begin{array}{l}24 / 35= \\
69 \%\end{array}$ & $\begin{array}{l}30 / 35= \\
86 \%\end{array}$ & $\begin{array}{l}30 / 35= \\
86 \%\end{array}$ & $\begin{array}{l}27 / 35= \\
77 \%\end{array}$ \\
\hline
\end{tabular}

ada cells: $\geq 20 \%$ immunoreactivity; light cells: $<20 \%$ immunoreactivity.

of primary mediastinal large B cell lymphoma (Table 3). The REL-negative cases did not exhibit any specific staining pattern for CD30, CD10, Bcl-2, and Bcl-6, although cases studied exhibited immunostaining for Bcl-6 (6/6) and were non-immunoreactive for CD10 (5/5; data not shown). In contrast, 5 of 30 cases of diffuse large B cell lymphoma (17\%) were immunoreactive for TRAF, and, in $2 / 5$ cases, at least 1 additional primary mediastinal large $\mathrm{B}$ cell lymphoma marker (Table 3).
Table 3 Summary of diffuse large B cell lymphoma immunostaining results ${ }^{\mathrm{a}}$

\begin{tabular}{|c|c|c|c|c|c|}
\hline Case no. & CD200 & CD23 & MAL & TRAF & REL \\
\hline \multicolumn{6}{|l|}{1} \\
\hline \multirow{2}{*}{\multicolumn{6}{|c|}{2}} \\
\hline & & & & & \\
\hline \multicolumn{6}{|l|}{3} \\
\hline \multicolumn{6}{|l|}{4} \\
\hline \multicolumn{6}{|l|}{5} \\
\hline \multicolumn{6}{|l|}{6} \\
\hline \multicolumn{6}{|l|}{7} \\
\hline \multicolumn{6}{|l|}{8} \\
\hline \multicolumn{6}{|l|}{9} \\
\hline \multicolumn{6}{|l|}{10} \\
\hline \multicolumn{6}{|l|}{11} \\
\hline \multicolumn{6}{|l|}{12} \\
\hline \multicolumn{6}{|l|}{13} \\
\hline \multicolumn{6}{|l|}{14} \\
\hline \multicolumn{6}{|l|}{15} \\
\hline \multicolumn{6}{|l|}{16} \\
\hline \multicolumn{6}{|l|}{17} \\
\hline \multicolumn{6}{|l|}{18} \\
\hline \multicolumn{6}{|l|}{19} \\
\hline \multicolumn{6}{|l|}{20} \\
\hline \multicolumn{6}{|l|}{21} \\
\hline \multicolumn{6}{|l|}{22} \\
\hline \multicolumn{6}{|l|}{23} \\
\hline \multicolumn{6}{|l|}{24} \\
\hline \multicolumn{6}{|l|}{25} \\
\hline \multicolumn{6}{|l|}{26} \\
\hline \multicolumn{6}{|l|}{27} \\
\hline \multicolumn{6}{|l|}{28} \\
\hline \multicolumn{6}{|l|}{29} \\
\hline \multicolumn{6}{|l|}{30} \\
\hline specificity & $\begin{array}{l}28 / 30= \\
93 \%\end{array}$ & $\begin{array}{l}28 / 30= \\
93 \%\end{array}$ & $\begin{array}{l}29 / 30= \\
97 \%\end{array}$ & $\begin{array}{l}23 / 30= \\
77 \%\end{array}$ & $\begin{array}{l}25 / 30= \\
83 \%\end{array}$ \\
\hline
\end{tabular}

a Dark cells: $\geq 20 \%$ immunoreactivity; light cells: $<20 \%$ immunoreactivity.

\section{Discussion}

CD200, an immunoglobulin superfamily membrane glycoprotein, is expressed in a range of hematopoietic neoplasms and is a useful immunophenotypic marker for immunohistochemical staining in diagnostic hematopathology. ${ }^{8,9}$ In addition, CD200 immunoreactivity can be assessed by flow-cytometric analysis in addition to immunohistochemical staining (Palumbo et $a l^{15}$ and Alapat et $a l^{16}$; Pillai et al, manuscript in preparation). A number of $\mathrm{B}$ cell-derived neoplasms are immunoreactive for CD200, including chronic lymphocytic leukemia/ small lymphocytic lymphoma, hairy cell leukemia, B lymphoblastic leukemia/lymphoma, multiple 
myeloma, and classical Hodgkin lymphoma., ${ }^{7,8}$ CD200 is useful in the differential diagnosis in a number of B cell-derived neoplasms, because it is not expressed in mantle cell lymphoma (vs chronic lymphocytic leukemia/small lymphocytic lymphoma), Burkitt lymphoma (vs B lymphoblastic leukemia/ lymphoma), or nodular lymphocyte predominant Hodgkin lymphoma (vs classical Hodgkin lymphoma; Dorfman and Shahsafaei ${ }^{8}$, Palumbo et al ${ }^{15}$; Pillai et al, in preparation). In a recent flow-cytometric study, we found that CD200 staining can differentiate hairy cell leukemia from hairy cell leukemia variant as well (Pillai et al, in preparation). In our initial study of CD200 expression in B cell lymphoproliferative disorders, we found that CD200 is expressed in primary mediastinal large B cell lymphoma but not in diffuse large B cell lymphoma. ${ }^{8}$ The current study substantiates the preliminary observation and suggests that CD200 is a useful marker for the differential diagnosis of large cell neoplasms of the mediastinum.

Our findings also are consistent with earlier studies on CD23, MAL, TRAF, and REL as markers useful for the distinction of primary mediastinal large B cell lymphoma from diffuse large B cell lymphoma. Calaminici et $a l^{11}$ found that $17 / 24$ cases $(70 \%)$ of primary mediastinal large B cell lymphoma were immunoreactive for CD23, while only $8 / 52$ cases of nodal diffuse large B cell lymphoma (15\%) and 4/48 cases of extranodal diffuse large B cell lymphoma (9\%) were CD200 positive (Table 1). This is similar to our finding of CD200 immunoreactivity in $69 \%$ of primary mediastinal large B cell lymphoma cases and $7 \%$ of diffuse large B cell lymphoma cases. In all, 21 of 33 cases of primary mediastinal large B cell lymphoma (70\%) and only $1 / 33$ cases of diffuse large B cell lymphoma (3\%) were initially found to immunoreactive for MAL. ${ }^{10}$ Similarly, we found that $86 \%$ of primary mediastinal large B cell lymphoma and $3 \%$ of diffuse large B cell lymphoma cases were MAL positive. Finally, TRAF and REL were found to be immunoreactive in $48 / 78$ cases $(62 \%)$ and $31 / 48$ cases $(65 \%)$ of primary mediastinal large B cell lymphoma, respectively, and 20/173 cases $(12 \%)$ and $28 / 160$ cases $(18 \%)$ of diffuse large B cell lymphoma. ${ }^{12}$ Similarly, we found that these markers were immunoreactive in 86 and $77 \%$ of primary mediastinal large B cell lymphoma cases, respectively, and 12 and $17 \%$ of diffuse large B cell lymphoma cases, respectively.

We previously found that the neoplastic cells in classical Hodgkin lymphoma and in primary mediastinal large B cell lymphoma, but not in nodular lymphocyte predominant Hodgkin lymphoma, are positive for CD200 in the majority of cases. ${ }^{8}$ Therefore, CD200 immunostaining should be helpful in the differential diagnosis of classical Hodgkin lymphoma vs nodular lymphocyte predominant Hodgkin lymphoma and diffuse large B cell lymphoma. However, additional markers are needed to discriminate classical Hodgkin lymphoma from primary mediastinal large $\mathrm{B}$ cell lymphoma on immunophenotypic grounds. Useful immunophenotypic markers for this purpose include CD15, a well-established marker of classical Hodgkin lymphoma that was found to be expressed in 17/20 cases $(85 \%)$ of classical Hodgkin lymphoma but none of 27 cases of primary mediastinal large B cell lymphoma in a recent study by Salama et al. ${ }^{18}$ That study also found that CD23, one of the immunophenotypic markers useful for distinguishing primary mediastinal large $\mathrm{B}$ cell lymphoma from diffuse large B cell lymphoma, was expressed in $23 / 27$ cases $(85 \%)$ of primary mediastinal large B cell lymphoma but only 2/18 cases (11\%) of classical Hodgkin lymphoma, in rare, scattered Reed-Sternberg cells. ${ }^{18}$ MAL, another primary mediastinal large B cell lymphoma marker, was found to be expressed in only $3 / 31$ cases of classical Hodgkin lymphoma, ${ }^{10}$ so it too may be useful for this application. In contrast, TRAF and REL fail to distinguish primary mediastinal large B cell lymphoma from classical Hodgkin lymphoma: $21 / 25$ cases (84\%) of classical Hodgkin lymphoma were found to be immunoreactive for TRAF and 23/25 cases (92\%) were immunoreactive for REL. ${ }^{19}$

Anti-CD200 targeted therapy may serve as a treatment for CD200-positive neoplasms, including chronic lymphocytic leukemia/small lymphocytic lymphoma. In an animal model of chronic lymphocytic leukemia, anti-CD200 antibody administration inhibits tumor growth, thought to be due to blocking of CD200 receptor-ligand interaction as well as elimination of activated T cells involved in immune mediated killing of neoplastic cells. ${ }^{20-22}$ ALXN6000, a humanized, murine-derived anti-human CD200 antibody is in clinical trial as a potential novel immunotherapeutic agent to treat chronic lymphocytic leukemia/small lymphocytic lymphoma and multiple myeloma (Kretz-Rommel and Bowdish $^{22}$; NCT00648739). If such therapy is found to be efficacious for these CD200-positive neoplasms, then it may be suitable to treat other CD200-positive B cell-derived neoplasms such as primary mediastinal large B cell lymphoma.

In conclusion, CD200 is a sensitive and specific marker for distinguishing primary mediastinal large B cell lymphoma from diffuse large B cell lymphoma in formalin-fixed, paraffin-embedded histologic sections. It sensitivity as a marker of primary mediastinal large B cell lymphoma exceeds that of other immunophenotypic markers studied and its staining specificity is comparable to that of other immunophenotypic markers of primary mediastinal large B cell lymphoma. CD200 is a useful addition to the armamentarium of immunophenotypic markers for the assessment of mediastinal lymphomas and large cell lymphomas at other sites of possible mediastinal origin. In addition, CD200 expression by the majority of cases of primary mediastinal large B cell lymphoma raises the possibility of a new immunotherapeutic treatment for this disease. 


\section{Disclosure/conflict of interest}

The authors declare no conflict of interest.

\section{References}

1 McCaughan GW, Clark MJ, Barclay AN. Characterization of the human homolog of the rat MRC OX-2 membrane glycoprotein. Immunogenetics 1987;25:329-335.

2 Wright GJ, Jones M, Puklavec MJ, et al. The unusual distribution of the neuronal/lymphoid cell surface CD200 (OX2) glycoprotein is conserved in humans. Immunology 2001;102:173-179.

3 Hoek RM, Ruuls SR, Murphy CA, et al. Downregulation of the macrophage lineage through interaction with OX-2 (CD200). Science 2000;290:1768-1771.

4 Wright GJ, Cherwinski H, Foster-Cuevas M, et al. Characterization of the CD200 receptor family in mice and humans and their interactions with CD200. J Immunol 2003;171:3034-3046.

5 Tonks A, Hills R, White P, et al. CD200 as a prognostic factor in acute myeloid leukemia. Leukemia 2007;21: 566-568.

6 Moreaux J, Hose D, Reme T, et al. CD200 is a new prognostic factor in multiple myeloma. Blood 2006;108:4194-4197.

7 Brunetti L, Di Noto R, Abate G, et al. CD200/OX2, a cell surface molecule with immune-regulatory function, is consistently expressed on hairy cell leukaemia neoplastic cells. Br J Haematol 2009;145:665-678.

8 Dorfman DM, Shahsafaei A. CD200 (OX-2 Membrane Glycoprotein) expression in B cell-derived neoplasms. Am J Clin Pathol 2010;134:726-733.

9 Dorfman DM, Shahsafaei A. CD200 (OX-2 Membrane Glycoprotein) is expressed by follicular helper T cells and in angioimmunoblastic T cell lymphoma. Am J Surg Pathol 2011;35:76-83.

10 Copie-Bergman C, Plonquet A, Alonso MA, et al. MAL expression in lymphoid cells: further evidence for MAL as a distinct molecular marker of primary mediastinal large B-cell lymphomas. Mod Pathol 2002;15:1172-1180.

11 Calaminici M, Piper K, Lee AM, et al. CD23 expression in mediastinal large B-cell lymphomas. Histopathology 2004;45:619-624.

12 Rodig SJ, Savage KJ, LaCasce AS, et al. Expression of TRAF1 and nuclear c-Rel distinguishes primary mediastinal large cell lymphoma from other types of diffuse large B-cell lymphoma. Am J Surg Pathol 2007;31:106-112.
13 Stein H, CHan JKC, Warnke RA, et al. Diffuse large B-cell lymphoma, not otherwise specified. Gaulard P, Stein H, Harris NL, et al. Primary mediastinal (thymic) large B-cell lymphoma. In: Swerdlow SH, Campo E, Harris NL, Jaffe ES, Pileri SA, Stein H, Thiele J, Vardiman JW (eds) WHO Classification of Tumours of Haematopoietic and Lymphoid Tissues LyonIARC Press; 2008, pp 233-237, 250-251.

14 Dorfman DM, Greisman HA, Shahsafaei A. Loss of expression of the WNT/beta-catenin-signaling pathway transcription factors lymphoid enhancer factor-1 (LEF-1) and $\mathrm{T}$ cell factor (TCF-1) in a subset of peripheral T cell lymphomas. Am J Pathol 2003;162: 1539-1544.

15 Palumbo GA, Parrinello N, Fargione G, et al. CD200 expression may help in differential diagnosis between mantle cell lymphoma and B-cell chronic lymphocytic leukemia. Leuk Res 2009;33:1212-1216.

16 Alapat D, Coviello-Malle J, Owens R, et al. Diagnostic usefulness and prognostic impact of CD200 expression in lymphoid malignancies and plasma cell myeloma. Am J Clin Pathol 2012;137:93-100.

17 Rosenwald, Wright G, Leroy K, et al. Molecular diagnosis of primary mediastinal B cell lymphoma identifies a clinically favorable subgroup of diffuse large B cell lymphoma related to Hodgkin lymphoma. J Exp Med 2003;198:851.

18 Salama ME, Mariappan MR, Inamdar $\mathrm{K}$, et al. The value of CD23 expression as an additional marker in distinguishing mediastinal (thymic) large B-cell lymphoma from Hodgkin lymphoma. Int J Surg Pathol 2010;18:121-128.

19 Rodig S, Savage KJ, Nguyen V, et al. TRAF1 expression and c-Rel activation are useful adjuncts in distinguishing classical Hodgkin lymphoma from a subset of morphologically or immunophenotypically similar lymphomas. Am J Surg Pathol 2005;29: 196-203.

20 Kretz-Rommel A, Qin F, Dakappagari N, et al. CD200 expression on tumor cells suppresses antitumor immunity: new approaches to cancer immunotherapy. J Immunol 2007;178:5595-5605.

21 Kretz-Rommel A, Qin F, Dakappagari N, et al. Blockade of CD200 in the presence or absence of antibody effector function: implications of anti-CD200 therapy. J Immunol 2008;180:699-705.

22 Kretz-Rommel A, Bowdish KS. Rationale for antiCD200 immunotherapy in B-CLL and other hematologic malignancies: new concepts in blocking immune suppression. Expert Opin Biol Ther 2008;8:5-15. 